\title{
Saturation Based Nonlinear PID control for Underwater Vehicles: Design, Stability Analysis and Experiments
}

\author{
J. Guerrero ${ }^{\mathrm{a}, *}$, J. Torres ${ }^{\mathrm{a}}$, V. Creuze $^{\mathrm{b}}$, A. Chemori ${ }^{\mathrm{b}}$, E. Campos $^{\mathrm{c}}$ \\ ${ }^{a}$ Automatic Control Department, CINVESTAV, Mexico CDMX, Mexico \\ ${ }^{b}$ LIRMM, Univ Montpellier, CNRS, Montpellier, France \\ ${ }^{c}$ CONACYT-Universidad del Istmo, Tehuantepec, Oaxaca, Mexico
}

\begin{abstract}
During sea missions, underwater vehicles are often exposed to changes in the parameters of their control systems and subject to external disturbances due to the influences of ocean currents. These issues make the design of a robust controller quite a challenging task. This paper focuses on the design of a nonlinear PID controller, based on a set of saturation functions for trajectory tracking on an underwater vehicle. The main feature of the proposed control law is that it preserves the advantages of robust control and remains easy to fine-tune in real applications. Using the Lyapunov concept, we prove the asymptotic stability of the closed-loop tracking system. The effectiveness and robustness of our proposed controller for trajectory tracking in depth and yaw dynamics is demonstrated through real-time experiments.

Keywords: Underwater vehicle, non-linear PID control, trajectory tracking, real-time experiments.
\end{abstract}

2010 MSC: 00-01, 99-00

\section{Introduction}

There are two main classes of Unmanned Underwater Vehicles (UUVs): Remotely Operated Vehicles (ROVs), and Autonomous Underwater Vehicles

\footnotetext{
Fully documented templates

* Corresponding author

Email address: jguerrero@ctrl.cinvestav.mx (J. Guerrero )
} 
(AUVs). Both require advanced controllers, as their dynamics are highly non 5 linear and they have to deal with unpredictable external disturbances, such as the ones generated by ocean currents or by the tether [1]. In the case of AUVs, all of the degrees of freedom (DoF) are controlled, while in the case of ROVs a part of the DoF are piloted by a human (shared control). Both classes require controllers and this paper will refer to Underwater Vehicles in general.

Proportional-Derivative (PD) and Proportional Integral Derivative (PID) controllers are the most commonly used techniques to control the position and orientation of commercial underwater vehicles, this is due to their design simplicity and their good performance, especially when some system parameters are unknown [2, 3, 4. However, it is well-known that when the plant's dynam15 ics is highly nonlinear, time-varying, or with significant time delays the PID controls performance is often degraded. The impact of these drawbacks can be reduced by using adaptive, saturated or nonlinear PD/PID strategies. Inspired by this problem, several advanced PD/PID control schemes for underwater vehicles have been proposed in previous literature and some of them are summarized

20

It is acknowledged that the PID control tuning process to obtain the best controller behavior can be time-consuming. Consequently, intelligent tuning and self-adjusting control parameter methodologies have been developed in recent years. In [5], a genetic algorithm was used to tune the gains of a fractional Following the same lines, a PID control was tuned using the Particle Swarm Optimization (PSO) method for setpoint regulation and trajectory tracking in diving and steering subsystems [6]. A Fuzzy Logic Controller (FLC) was used with the PID algorithm to tune its gains adaptively. For example, in [7], a so decoupled Adaptive Fuzzy PID Controller (AFPIDC) for trajectory tracking in heading and depth of an AUV was proposed. In this work, the adaption law is composed of two elements, the initial constant control gains, given by the designer, and the time-varying incremental gains which depend on the tracking error and its ratio. The incremental gain is adjusted by fuzzy rules derived from 
the expert's knowledge. Based on simulation results, the performance of the AFPIDC is superior to nominal PID design during tracking trajectory tests. Similar methodologies, using fuzzy logic to improve the PID controller for path following or to demonstrate its robustness with respect to external disturbances can be found in [8, 9]. Finally, inverse optimal PID control applied to a selftuning controller for an AUV, modeled as a nonlinear autoregressive moving average model with exogenous inputs was proposed in [10].

The Active Disturbance Rejection Controller (ADRC) can estimate the influence of the external disturbances such as ocean currents or wave effects over an AUV. On the one hand, in paper [1, an adaptive DOB control (ADOB) 45 for set point regulation and trajectory tracking problems on the 6 degrees of freedom of ODIN AUV was proposed. In this work, the proposed controller was designed for a known nominal model, where the external disturbances and modeling errors were estimated through the DOB method. Then, a regressorfree adaptive control law was adopted to provide robustness to the DOB control towards uncertainties in the system model. The effectiveness of the proposed methodology was shown through real-time experiments on the $\mathrm{x}-\mathrm{y}-\mathrm{z}$ dynamics, while the AUV's orientation was kept stable (i.e., $\phi=\theta=\psi=0$ ). From these results, we can observe that the ADOB algorithm improves the performance of the PID controller considerably under constant external disturbances and pa${ }_{55}$ rameter uncertainties. On the other hand, in [11, the DOB method was applied to the PID control of an AUV based on the frequency analysis approach. In [12], a diving ADRC has been proposed to deal with the high nonlinearity, strong coupling and time-varying features in the AUV system.

It is worth noting that, during sea missions, an AUV can be disturbed by ocean currents or subject to unknown objects sticking to the submarine body which suddenly changes its physical parameters. To overcome this problem, adaptive controllers can be used as a suitable solution to control AUVs. The main feature of an adaptive controller lies in its ability to update the control gains based on the changes in vehicle dynamics and external disturbances. As 65 an example of this methodology, an adaptive PD controller for setpoint regula- 
tion was proposed in [13]. The designed controller needed only the knowledge of the vector of gravitational and buoyancy forces. The control law consists of a PD plus buoyancy compensation (PD+) with an adaptive term that estimates and compensates parameter uncertainties and external disturbances. The behavior of the adaptive controller was validated through simulations and real-time experiments for setpoint regulation in $(x, y, z, \psi)$ dynamics. Based on the obtained experimental results, it can be observed that the adaptive control has a better performance in depth dynamics than the $\mathrm{PD}$, but the behavior of both methodologies is almost the same for $(x, y, \psi)$ dynamics. Also, following the same methodology, an adaptive PD controller for a region reaching controller was proposed in [14. We can compare this to [13, which is based on a saturated PD control instead of a linear PD law. Although the effectiveness of the proposed controller was only shown in simulations.

In practical applications, it can be observed that a standard PID control design can be improved by bounding its signal [15]. Consequently, several nonlinear bounded PID controllers have been proposed. For instance, in [16, a model reference adaptive (MRAC) PID control structure with an anti-windup (AW) compensator for pitch trajectory tracking of the REMUS AUV was proposed. It was demonstrated that adding the AW compensator improves the nominal 85 adaptive control. The AW term is obtained by solving a linear Ricatti equation. Simulation results show the improvements of the proposed controller over the nominal MRAC in terms of external disturbances rejection and saturation of the AUV's actuators. Another version taking into account the saturation of the AUV's actuators, resulting in a $\mu$ modified adaptive controller was proposed in 17. Also, a dual-loop variable-structure PID (VSPID) controller with AW term for controlling the surge and sway dynamics of an AUV is proposed in [18. Experimental results show that the VSPID with AW reduces the overshoot as well as the settling time compared to the nominal VSPID. Finally, inspired by the works of [19] and 20, a nonlinear PD and PD+ controllers for trajectory tracking on depth and yaw dynamics of an AUV has been proposed in [15]. In this work, the authors introduced a whole set of nonlinear functions to improve the 
PD controller. Real-time experiments on the L2ROV vehicle demonstrate the effectiveness and robustness of the proposed control law. Indeed, an improved performance of the proposed controller for trajectory tracking in yaw dynamics is demonstrated. However, the performance of the controller for depth trajectory tracking is reduced when the system's parameters are subject to uncertainties.

In summary, on the one hand, fuzzy approaches [7] and intelligent algorithms such as PSO and AG (see [5], [6]), which are used to tune the PID control, can be useful to obtain good performance from the controller. On the other hand, the disturbance estimation made by ADRC can provide robustness to the PID, as seen in the experiments shown in references [1, [11, ,12. However, based on the experimental results of these works, the robustness improvement of this methodology towards parameter uncertainties or external disturbances is not clear. Furthermore, the main ADRC drawback is the tedious task of tuning numerous parameters. Concerning the adaptive controllers [13, 14, their main advantages are the self-adjustment of gains and the fact that only partial information about the vehicle's mathematical model is required. However, the low rate of gains adjustment and the overestimation of feedback gains remains a drawback to this method. Continuing, the MRAC with the AW term can improve PID performance, as shown in the simulation results of study [16. Nevertheless, the proposed methodology requires computing the Ricatti equation online, which could be difficult. Finally, the introduction of saturation functions in the gains of the PD controller improves its performance, as one can see in the experimental results of work [15]. However, this methodology is not robust enough to encompass large and persistent parameter uncertainties. Taking into account this drawback, in this paper, a nonlinear PID controller is proposed to overcome the shortcoming of the previous algorithm introduced in [15]. The main contributions of the actual work are summarized as follows:

(i) A whole range of nonlinear functions to improve the PID controller are proposed.

(ii) The stability analysis of the proposed controller is formalized based on 
Lyapunov design.

(iii) External disturbance rejection and robustness towards parameter uncertainties are demonstrated through real-time experiments.

130 (Society of Naval Architects and Marine Engineers) notation 23] and the representation introduced by [3] can be written as follows:

$$
\begin{aligned}
M \dot{\nu}+C(\nu) \nu+D(\nu) \nu+g(\eta) & =\tau+w_{e}(t) \\
\dot{\eta} & =J(\eta) \nu
\end{aligned}
$$

where $\nu=[u, v, w, p, q, r]^{T}$ is the vector of velocity in the body-fixed frame and $\eta=[x, y, z, \phi, \theta, \psi]^{T}$ represents the vector of position and orientation in the 150 earth-fixed frame. $J(\eta) \in \mathbb{R}^{6 \times 6}$ is the transformation matrix between the inertial frame and the body-fixed frame. $M \in \mathbb{R}^{6 \times 6}$ is the inertia matrix where the 


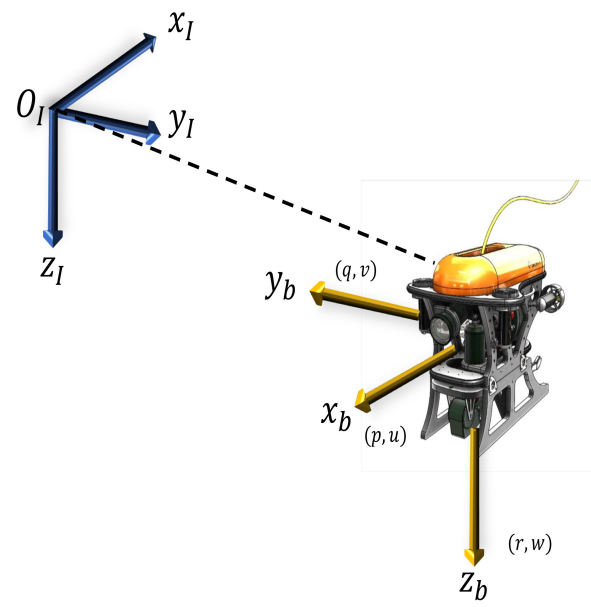

Figure 1: The underwater vehicle with the inertial-fixed frame $\left(O_{I}, x_{I}, y_{I}, z_{I}\right)$ and the bodyfixed frame $\left(O_{b}, x_{b}, y_{b}, z_{b}\right)$.

effects of added mass are considered, $C(\nu) \in \mathbb{R}^{6 \times 6}$ is the Coriolis-centripetal matrix, $D(\nu) \in \mathbb{R}^{6 \times 6}$ representing the hydrodynamic damping matrix also including the effects of added-mass, $g(\eta) \in \mathbb{R}^{6}$ is the vector of gravitational/buoyancy forces and moments. Finally, $\tau \in \mathbb{R}^{6}$ is the control input vector acting on the underwater vehicle and $w_{e}(t) \in \mathbb{R}^{6}$ represents the vector of external disturbances.

The above formulation for underwater vehicle dynamics is expressed in the body-fixed frame and can be transformed to the earth-fixed frame based on the kinematic transformations of the state variables and the model parameters as follows:

$$
\begin{aligned}
M_{\eta}(\eta) & =J^{-T}(\eta) M J^{-1}(\eta) \\
C_{\eta}(\nu, \eta) & =J^{-T}(\eta)\left[C(\nu)-M J^{-1}(\eta) \dot{J}(\eta)\right] J^{-1}(\eta) \\
D_{\eta}(\nu, \eta) & =J^{-T}(\eta) D(\nu) J^{-1}(\eta) \\
g_{\eta}(\eta) & =J^{-T}(\eta) g(\eta) \\
\tau_{\eta}(\eta) & =J^{-T}(\eta) \tau
\end{aligned}
$$

Based on these equations, the dynamics (1) can therefore be rewritten in the 
earth-fixed frame as:

$$
M_{\eta}(\eta) \ddot{\eta}+C_{\eta}(\nu, \eta) \dot{\eta}+D_{\eta}(\nu, \eta) \dot{\eta}+g_{\eta}(\eta)=\tau_{\eta}(\eta)+w_{\eta}(t)
$$

For a deeper description of the model, the reader may refer to 3,24 .

\section{Proposed Nonlinear PID Controller}

In this section, a nonlinear PID controller based on saturation functions with variable parameters is introduced. The design of the controller is focused on both setpoint regulation, as well as trajectory tracking. The stability analysis of the resulting closed-loop system for both cases is explained in detail. Let us consider the underwater vehicle mathematical model (2), and the following control law

$$
\tau=J^{T}\left[M_{\eta}(\eta) \ddot{\eta}_{d}+C_{\eta}(\nu, \eta) \dot{\eta}_{d}+D_{\eta}(\nu, \eta) \dot{\eta}_{d}+g(\eta)-\tau_{P I D}\right]
$$

where the PID controller is defined as follows:

$$
\tau_{P I D}=K_{p} e(t)+K_{i} \int_{0}^{t} e(s) d s+K_{d} \frac{d e(t)}{d t}
$$

The feedback gains are defined as $K_{p}=\operatorname{diag}\left(k_{p 1}, k_{p 2}, \cdots, k_{p 6}\right)>0, K_{i}=$ $\operatorname{diag}\left(k_{i 1}, k_{i 2}, \cdots, k_{i 6}\right)>0$, and $K_{d}=\operatorname{diag}\left(k_{d 1}, k_{d 2}, \cdots, k_{d 6}\right)>0$, where $k_{p j}, k_{i j}$ and $k_{d j}$ are positive constants for all $j=1, \cdots, 6$. The error is expressed as $e(t)=\left[e_{1}(t), e_{2}(t), \cdots, e_{6}(t)\right]^{T}=\eta(t)-\eta_{d}(t)$, with $\eta_{d}(t)$ the desired trajectory vector.

In order to improve the performance of the closed-loop system, we propose introducing a saturation function $\sigma_{\bar{b}}(h)$ defined as:

$$
\sigma_{\bar{b}}(h)= \begin{cases}\bar{b} & \text { if } h>\bar{b} \\ h & \text { if }|h| \leq \bar{b} \\ -\bar{b} & \text { if } h<-\bar{b}\end{cases}
$$

where $\bar{b}$ is a positive constant, and $h$ represents a linear function. In this paper, the terms to which this saturation will be applied are the tracking error, its 
integral, and its time derivative. Then, if we consider this saturation with the previous control law, we obtain the following nonlinear PID (NLPID) controller:

$$
\tau_{N L P I D}=\sigma_{\bar{b} p}\left[K_{p} e(t)\right]+\sigma_{\bar{b} i}\left[K_{i} \int_{0}^{t} e(s) d s\right]+\sigma_{\bar{b} d}\left[K_{d} \frac{d e(t)}{d t}\right]
$$

where

$$
\begin{array}{r}
\sigma_{\bar{b} p}\left[K_{p} e(t)\right]=\left[\begin{array}{cccc}
u_{p 1} & 0 & \cdots & 0 \\
0 & u_{p 2} & \cdots & 0 \\
\vdots & \vdots & \ddots & \vdots \\
0 & 0 & \cdots & u_{p 6}
\end{array}\right] \\
\sigma_{\bar{b} i}\left[K_{i} \int_{0}^{t} e(s) d s\right]=\left[\begin{array}{cccc}
u_{i 1} & 0 & \cdots & 0 \\
0 & u_{i 2} & \cdots & 0 \\
\vdots & \vdots & \ddots & \vdots \\
0 & 0 & \cdots & u_{i 6}
\end{array}\right] \\
\sigma_{\bar{b} d}\left[K_{d} \frac{d e(t)}{d t}\right]=\left[\begin{array}{cccc}
u_{d 1} & 0 & \cdots & 0 \\
0 & u_{d 2} & \cdots & 0 \\
\vdots & \vdots & \ddots & \vdots \\
0 & 0 & \cdots & u_{d 6}
\end{array}\right]
\end{array}
$$

$170 \quad$ with $u_{p_{j}}=\sigma_{\bar{b} p_{j}}\left[k_{p j} e_{j}(t)\right], u_{i_{j}}=\sigma_{\bar{b} i_{j}}\left[k_{i j} \int_{0}^{t} e_{j}(s) d s\right]$, and $u_{d_{j}}=\sigma_{\bar{b} d_{j}}\left[k_{d j} \frac{d e_{j}(t)}{d t}\right]$ for all $j=1, \cdots, 6$.

Without loss of generality, let us now consider the scalar case:

$$
\tau_{N L P I D 1}=\sigma_{\bar{b} p_{1}}\left[k_{p 1} e_{1}(t)\right]+\sigma_{\bar{b} i_{1}}\left[k_{i 1} \int_{0}^{t} e_{1}(s) d s\right]+\sigma_{\bar{b} d_{1}}\left[k_{d 1} \frac{d e_{1}(t)}{d t}\right]
$$

The above equation can be rewritten in a compact form as follows:

$$
\tau_{N L P I D 1}=\sum_{n=1}^{3} u_{n}
$$

where $u_{n}=\sigma_{\bar{b}_{n}}\left(k_{n} h_{n}\right)$ represents the saturation function, with $\bar{b}_{1}=\bar{b}_{p 1}, \bar{b}_{2}=$ $\bar{b}_{i 1}, \bar{b}_{3}=\bar{b}_{d 1}$, and the controller feedback gains $k_{1}=k_{p 1}, k_{2}=k_{i 1}, k_{3}=k_{d 1}$. The error term, its integral and its time derivative are represented by $h_{1}, h_{2}$, 
and $h_{3}$, respectively. Then, from equation (5), $u_{n}$ can be rewritten as:

$$
u_{n}= \begin{cases}\bar{b}_{n} & \text { if } k_{n} h_{n}>\bar{b}_{n} \\ k_{n} h_{n} & \text { if }\left|k_{n} h_{n}\right| \leq \bar{b}_{n} \\ -\bar{b}_{n} & \text { if } k_{n}<-\bar{b}_{n}\end{cases}
$$

In the above equation, we can observe that the linear function $k_{n} h_{n}$ is saturated by $\left|h_{n}\right|=\bar{b}_{n} / k_{n}$. Let us define $d_{n}$ as:

$$
d_{n}=\bar{b}_{n} / k_{n}
$$

Then, 12 can be rewritten as follows:

$$
u_{n}= \begin{cases}\operatorname{sgn}\left(h_{n}\right) \bar{b}_{n} & \text { if }\left|h_{n}\right|>d_{n} \\ \bar{b}_{n} \bar{d}_{n}^{-1} h_{n} & \text { if }\left|h_{n}\right| \leq d_{n}\end{cases}
$$

where tuning parameters of the controller are $b_{n}$ and $d_{n}$, for $n=1,2,3$. Moreover, considering that:

$$
\operatorname{sgn}\left(h_{n}\right) \bar{b}_{n}=h_{n} \operatorname{sgn}\left(h_{n}\right) \bar{b}_{n} \bar{h}_{n}^{-1}
$$

which can be simplified as:

$$
\operatorname{sgn}\left(h_{n}\right) \bar{b}_{n}=\left|h_{n}\right| \bar{b}_{n} \bar{h}_{n}^{-1}
$$

and considering that $\left|h_{n}\right| h_{n}^{-1}=\left|h_{n}\right|^{-1} h_{n}$, equation (14) can be rewritten as follows:

$$
u_{n}= \begin{cases}\bar{b}_{n}\left|h_{n}\right|^{-1} h_{n} & \text { if }\left|h_{n}\right|>d_{n} \\ \bar{b}_{n} \bar{d}_{n}^{-1} h_{n} & \text { if }\left|h_{n}\right| \leq d_{n}\end{cases}
$$

Consequently, the control law 10 can be rewritten as follows:

$$
\tau_{N L P I D 1}=u_{1}+u_{2}+u_{3}=k_{p 1}(\cdot) e_{1}(t)+k_{i 1}(\cdot) \int_{0}^{t} e_{1}(s) d s+k_{d 1}(\cdot) \dot{e}_{1}(t)
$$


with

$$
\begin{aligned}
& k_{p 1}(\cdot)= \begin{cases}\bar{b}_{p 1}|e(t)|^{-1} & \text { if }\left|e_{1}(t)\right|>d_{p 1} \\
\bar{b}_{p 1} \bar{d}_{p 1}^{-1} & \text { if }\left|e_{1}(t)\right| \leq d_{p 1}\end{cases} \\
& k_{i 1}(\cdot)= \begin{cases}\bar{b}_{i 1}\left|\int_{0}^{t} e_{1}(s)\right|^{-1} d s & \text { if }\left|\int_{0}^{t} e_{1}(s)\right| d s>d_{i 1} \\
\bar{b}_{i 1} \bar{d}_{i 1}^{-1} & \text { if }\left|\int_{0}^{t} e_{1}(s)\right| d s \leq d_{i 1}\end{cases} \\
& k_{d 1}(\cdot)= \begin{cases}\bar{b}_{d 1}\left|\dot{e}_{1}(t)\right|^{-1} & \text { if }\left|\dot{e}_{1}(t)\right|>d_{d 1} \\
\bar{b}_{d 1} \bar{d}_{d 1}^{-1} & \text { if }\left|\dot{e}_{1}(t)\right| \leq d_{d 1}\end{cases}
\end{aligned}
$$

The advantage of this formulation is that the forces and torques are bounded by the saturation parameters $\bar{b}_{p 1}, \bar{b}_{i 1}$ and $\bar{b}_{d 1}$. Consequently, the saturation of the control input is guaranteed. However, some cases may require slightly larger forces and torques to correct the system errors, that is why we propose to change the saturation value $\bar{b}_{n}$ in equation $(18)$ as follows:

$$
\bar{b}_{n}= \begin{cases}b_{n}\left|h_{n}\right|^{\mu_{n}} & \text { if }\left|h_{n}\right|>d_{n} \\ b_{n}\left|d_{n}\right|^{\mu_{n}} & \text { if }\left|h_{n}\right| \leq d_{n}\end{cases}
$$

$b_{n}$ being a positive constant, and $\mu_{n} \in[0,1]$. Now, introducing equations 22 into (17), leads to:

$$
u_{n}= \begin{cases}\bar{b}_{n}\left|h_{n}\right|^{\mu_{n}}\left|h_{n}\right|^{-1} h_{n} & \text { if }\left|h_{n}\right|>d_{n} \\ \bar{b}_{n}\left|d_{n}\right|^{\mu_{n}} \bar{d}_{n}^{-1} h_{n} & \text { if }\left|h_{n}\right| \leq d_{n}\end{cases}
$$

Note that the shape of the function $u_{n}$ depends on the selected parameter value $\mu_{n}$ as illustrated in Figure 2. Consequently, the nonlinear PID control law based on saturation functions with variable parameters can be expressed as:

$$
\tau_{N L P I D j}=k_{p j}(\cdot) e_{j}(t)+k_{i j}(\cdot) \int_{0}^{t} e_{j}(s) d s+k_{d j}(\cdot) \dot{e}_{j}(t)
$$




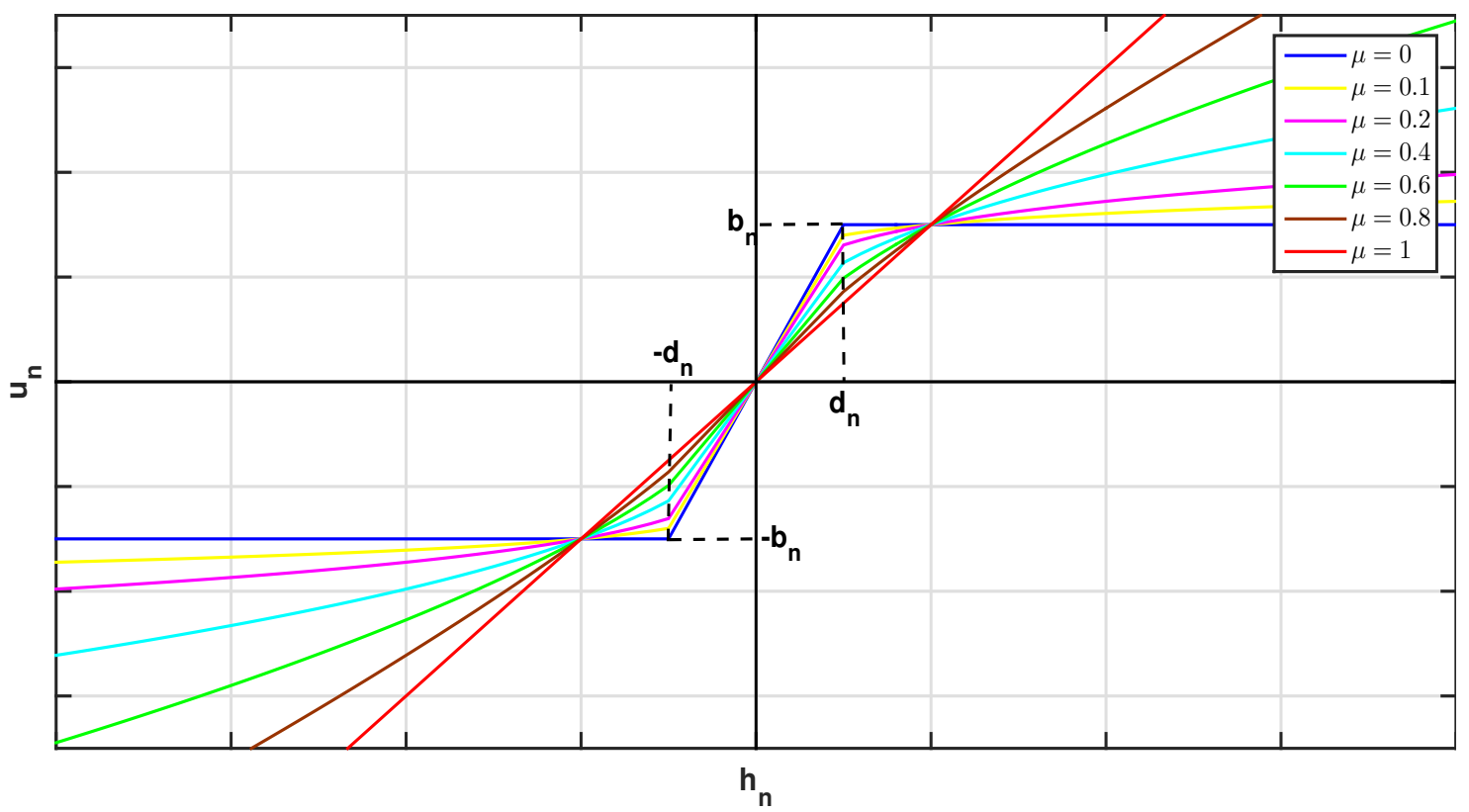

Figure 2: Illustration of the saturation function $u_{n}$ for different values of the parameter $\mu_{n}$.

with:

$$
\begin{aligned}
& k_{p j}(\cdot)= \begin{cases}\bar{b}_{p j}\left|e_{j}(t)\right|^{\left(\mu_{p j}-1\right)} & \text { if }\left|e_{j}(t)\right|>d_{p j} \\
\bar{b}_{p j} \bar{d}_{p j}^{\left(\mu_{p j}-1\right)} & \text { if }\left|e_{j}(t)\right| \leq d_{p j}\end{cases} \\
& k_{i j}(\cdot)= \begin{cases}\bar{b}_{i j}\left|\int_{0}^{t} e_{j}(s)\right|^{\left(\mu_{i j}-1\right)} d s & \text { if }\left|\int_{0}^{t} e_{j}(s)\right| d s>d_{i j} \\
\bar{b}_{i j} \bar{d}_{i j}^{\left(\mu_{i j}-1\right)} & \text { if }\left|\int_{0}^{t} e_{j}(s)\right| d s \leq d_{i j}\end{cases} \\
& k_{d j}(\cdot)= \begin{cases}\bar{b}_{d j}\left|\dot{e}_{j}(t)\right|^{\left(\mu_{d j}-1\right)} & \text { if }\left|\dot{e}_{j}(t)\right|>d_{d j} \\
\bar{b}_{d j} \bar{d}_{d j}^{\left(\mu_{d j}-1\right)} & \text { if }\left|\dot{e}_{j}(t)\right| \leq d_{d j}\end{cases}
\end{aligned}
$$

$\forall \mu_{p j}, \mu_{i j}, \mu_{d j} \in[0,1]$ 


\subsection{Stability Analysis}

Let us consider the nonlinear PID controller given as 24) and define the gain matrices as follows:

$$
\begin{aligned}
K_{p}^{\prime}(\cdot) & =K_{p}(\cdot)-\frac{1}{\alpha} K_{i}(\cdot) \\
K_{i}^{\prime}(\cdot) & =\frac{1}{\alpha} K_{i}(\cdot) \\
\zeta & =\int_{0}^{t}[\alpha e(s)+\dot{e}(s)] d s
\end{aligned}
$$

with $K_{p}(\cdot)=\operatorname{diag}\left(k_{p 1}(\cdot), k_{p 2}(\cdot), k_{p 3}(\cdot), k_{p 4}(\cdot), k_{p 5}(\cdot), k_{p 6}(\cdot)\right)>0$ and $K_{i}(\cdot)=$ $\operatorname{diag}\left(k_{i 1}(\cdot), k_{i 2}(\cdot), k_{i 3}(\cdot), k_{i 4}(\cdot), k_{i 5}(\cdot), k_{i 6}(\cdot)\right)>0$.

The complete control law (3) taking into account the nonlinear PID (24), can be rewritten in the following form:

$$
\tau=J^{T}(\eta)\left[M_{\eta}(\eta) \ddot{\eta}_{d}+C_{\eta}(\nu, \eta) \dot{\eta}_{d}+D_{\eta}(\nu, \eta) \dot{\eta}_{d}+g(\eta)-K_{p}^{\prime}(\cdot) e-K_{d}(\cdot) \dot{e}-K_{i}^{\prime}(\cdot) \zeta\right]
$$

with $K_{d}(\cdot)=\operatorname{diag}\left(k_{d 1}(\cdot), k_{d 2}(\cdot), k_{d 3}(\cdot), k_{d 4}(\cdot), k_{d 5}(\cdot), k_{d 6}(\cdot)\right)>0$.

Injecting the PID control (31) into dynamic system (2), leads to the following closed loop system:

$$
\ddot{e}=M_{\eta}(\eta)^{-1}\left[-C_{\eta}(\nu, \eta) \dot{e}-D_{\eta}(\nu, \eta) \dot{e}-K_{p}^{\prime}(\cdot) e-K_{i}^{\prime}(\cdot) \zeta-K_{d}(\cdot) \dot{e}\right]
$$

which can be rewritten in an augmented state form as follows:

$$
\frac{d}{d t}\left[\begin{array}{c}
e \\
\dot{e} \\
\zeta
\end{array}\right]=\left[\begin{array}{c}
\dot{e} \\
M_{\eta}(\eta)^{-1}\left[-C_{\eta}(\nu, \eta) \dot{e}-D_{\eta}(\nu, \eta) \dot{e}-K_{p}^{\prime}(\cdot) e-K_{i}^{\prime}(\cdot) \zeta-K_{d}(\cdot) \dot{e}\right] \\
\alpha e+\dot{e}
\end{array}\right]
$$

Remark 1. Note that the origin of the state space model is a unique equilibrium point. 
Remark 2. The main challenge is to find a Lyapunov candidate function that will allow us to take into account the auxiliary state variable $\zeta$ as it appears in Eq. 33. As we will see below, this is not a trivial extension of the PD controller case, see 14.

Consider the following Lyapunov Candidate Function:

$$
\begin{aligned}
V(e, \dot{e}, \zeta)= & \frac{1}{2} \dot{e}^{T} M_{\eta}(\eta) \dot{e}+\alpha e^{T} M_{\eta}(\eta) \dot{e}+\frac{\alpha}{2} e^{T} D_{\eta} e \\
& +\int_{0}^{e} \xi^{T} K_{p}^{\prime}(\xi) d \xi+\int_{0}^{\zeta} \xi^{T} K_{i}^{\prime}(\xi) d \xi+\alpha \int_{0}^{e} \xi^{T} K_{d}(\xi) d \xi
\end{aligned}
$$

which can be rewritten as follows:

$$
\begin{aligned}
V(e, \dot{e}, \zeta)= & \frac{1}{2}[\dot{e}+\alpha e]^{T} M_{\eta}(\eta)[\dot{e}+\alpha e]+\frac{\alpha}{2} e^{T}\left[D_{\eta}-\alpha M_{\eta}(\eta)\right] e+ \\
& +\int_{0}^{e} \xi^{T} K_{p}^{\prime}(\xi) d \xi+\int_{0}^{\zeta} \xi^{T} K_{i}^{\prime}(\xi) d \xi+\alpha \int_{0}^{e} \xi^{T} K_{d}(\xi) d \xi
\end{aligned}
$$

To prove that the Lyapunov candidate function is a positive definite and radially unbounded, let us consider the following:

$$
\begin{aligned}
\int_{0}^{e} \xi^{T} K_{p}(\xi) d \xi= & \int_{0}^{e_{1}} \xi_{1} k_{p 1}\left(\xi_{1}\right) d \xi_{1}+\int_{0}^{e_{2}} \xi_{2} k_{p 2}\left(\xi_{2}\right) d \xi_{2}+ \\
& +\int_{0}^{e_{3}} \xi_{3} k_{p 3}\left(\xi_{3}\right) d \xi_{3}+\cdots+\int_{0}^{e_{n}} \xi_{n} k_{p n}\left(\xi_{n}\right) d \xi_{n}
\end{aligned}
$$

where the inequality

$$
e_{j} k_{p j}(\cdot) \geq \alpha_{j}\left(\left|e_{j}\right|\right)
$$

is satisfied with class- $K$ functions

$$
\alpha_{j}\left(\left|e_{j}\right|\right)= \begin{cases}\frac{b_{j}\left|e_{j}\right|^{\mu_{p j}} e_{j}}{a+\left|e_{j}\right|} & \text { if }\left|e_{j}\right|>d_{j} \\ \frac{b_{j}\left|d_{j}\right|^{\mu_{p j}} e_{j}}{a+d_{j}} & \text { if }\left|e_{j}\right| \leq d_{j}\end{cases}
$$

with $b_{p j}>b_{j}, a>0$ and $d_{p j}<d_{j}$. Then, according to Lemma 2 from [8], we can deduce that:

$$
\int_{0}^{e} \xi^{T} K_{p}(\xi) d \xi>0 \quad \forall e \neq 0 \in \mathbb{R}^{n}
$$

and

$$
\int_{0}^{e} \xi^{T} K_{p}(\xi) d \xi \rightarrow \infty \quad \text { as } \quad\|e\| \rightarrow \infty
$$


Note that by following the same arguments, we can conclude that the next conditions are accomplished:

$$
\begin{array}{ll}
\int_{0}^{\zeta} \xi^{T} K_{i}(\xi) d \xi>0 & \forall \zeta \neq 0 \in \mathbb{R}^{n} \\
\int_{0}^{e} \xi^{T} K_{d}(\xi) d \xi>0 & \forall e \neq 0 \in \mathbb{R}^{n}
\end{array}
$$

and

$$
\begin{aligned}
& \int_{0}^{\zeta} \xi^{T} K_{i}(\xi) d \xi \rightarrow \infty \text { as }\|\zeta\| \rightarrow \infty \\
& \int_{0}^{e} \xi^{T} K_{d}(\xi) d \xi \rightarrow \infty \text { as }\|e\| \rightarrow \infty
\end{aligned}
$$

The term $1 / 2[\dot{e}+\alpha e]^{T} M_{\eta}(\eta)[\dot{e}+\alpha e]$ is positive definite because the matrix $M_{\eta}(\eta)$ is positive definite. Finally, the matrix $D_{\eta}-\alpha M_{\eta}(\eta)$ is positive definite since:

$$
\frac{D_{\eta i}}{\sum_{j=1}^{n} \max _{\eta}\left|M_{\eta i j}(\eta)\right|}>\alpha
$$

where $M_{\eta i j}(\eta)$ stands for the element of matrix $M_{\eta}(\eta)$ placed at row $i$ and column $j$. Therefore, the Lyapunov function candidate $V(e, \dot{e}, \zeta)$ is positive definite and radially unbounded.

The time derivative of the Lyapunov candidate function, step by step, is given as:

$$
\begin{aligned}
\dot{V}(e, \dot{e}, \zeta)= & \dot{e}^{T} M_{\eta} \ddot{e}+\frac{1}{2} \dot{e}^{T} \dot{M}_{\eta} \dot{e}+\alpha \dot{e}^{T} M_{\eta} \dot{e}+\alpha e^{T} \dot{M}_{\eta} \dot{e}+\alpha e^{T} M_{\eta} \ddot{e} \\
& +\alpha e^{T} K_{d}(\cdot) \dot{e}+e^{T} K_{p}^{\prime}(\cdot) \dot{e}+\zeta^{T} K_{i}^{\prime}(\cdot) \dot{\zeta}+\alpha e^{T} D_{\eta} \dot{e} \\
= & \frac{1}{2} \dot{e}^{T}\left[\dot{M}_{\eta}-2 C_{\eta}\right] \dot{e}-\dot{e}^{T} D_{\eta} \dot{e}-\dot{e}^{T} K_{d}(\cdot) \dot{e}+\alpha \dot{e}^{T} M_{\eta} \dot{e}+\alpha e^{T} \dot{M}_{\eta} \dot{e} \\
& -\alpha e^{T} C_{\eta} \dot{e}-\alpha e^{T} K_{p}^{\prime}(\cdot) e
\end{aligned}
$$

From the assumption that the vehicle is moving at low speed, we can assume that $\dot{M}_{\eta}=0, C_{\eta}(\nu, \eta)$ is skew symmetric and $D(\nu, \eta)>0$, then:

$$
\dot{V}(e, \dot{e})=-\dot{e}^{T}\left[K_{d}(\cdot)+D_{\eta}-\alpha M_{\eta}\right] \dot{e}-\alpha e^{T} K_{p}^{\prime}(\cdot) e
$$

From 45 it is possible to observe that the term on the right hand side is negative because $\alpha>0$ and $K_{p}^{\prime}(\cdot)>0$. The first term $K_{d}(\cdot)+D_{\eta}-\alpha M_{\eta}$ will 
be positive if condition (44) is satisfied. Finally, we can conclude that $\dot{V}$ is negative semidefinite and based on the LaSalle invariance principle it is possible to ensure asymptotic stability.

\section{Real-Time Experimental Results}

To demonstrate the feasibility and efficiency of our proposed control solution, we applied the control algorithm to Leonard (Figure 3), an underwater vehicle developed at the LIRMM (CNRS/University of Montpellier, France). Leonard is a tethered underwater vehicle that measures $75 \times 55 \times 45 \mathrm{~cm}$ and weighs 28 $\mathrm{kg}$. The propulsion system of this vehicle consists of six thrusters to obtain a fully actuated system.

The underwater robot is controlled by a laptop computer, with CPU Intel Core i7-3520M 2.9 GHz, 8GB of RAM memory. The computer runs under Windows 7 operating system and the control software is developed using Visual $\mathrm{C}++2010$. The computer receives the data from the robot's sensors (depth, IMU), computes the control laws and sends input signals to the propellers. These actuators are controlled by Syren 25 Motor Drivers. The main features of this vehicle are summarized in Table 1. It is worth noting that this vehicle has two operation modes, we can use it as a ROV for sea exploration missions or we can program tasks to be performed autonomously. In our paper, we focus in the latter case. All the trajectory tracking tests were performed as an AUV. The control algorithm was experimentally tested in the $4 \times 4 \times 1.2 \mathrm{~m}$ pool of the LIRMM. Although, the proposed control law given by (24) is designed for the whole system of six degrees of freedom, the real-time experiments conducted in this work concern only depth and yaw. The main objective of the designed control law is to robustly track a desired reference trajectory in depth and yaw in presence of parameter uncertainties and/or external disturbances. Real-time experiments can be seen at https://www.youtube.com/watch?v=lkiYr0v7H7c. 
Table 1: Main Features of the underwater vehicle

\begin{tabular}{|c|c|}
\hline Mass & $28 \mathrm{~kg}$ \\
\hline Buoyancy & $9 N$ \\
\hline Dimensions & $75 \times 55 \times 45 \mathrm{~cm}$ \\
\hline Maximal depth & $100 \mathrm{~m}$ \\
\hline Thrusters & 6 Seabotix BTD150 \\
\hline Power & $48 \mathrm{~V}-600 \mathrm{~W}$ \\
\hline \multirow[t]{5}{*}{ Attitude Sensor } & Sparkfun Arduimu V3 \\
\hline & Invensense MPU-6000 MEMS 3-axis gyro \\
\hline & and accelerometer \\
\hline & 3-axis I2C magnetometer HMC-5883L \\
\hline & Atmega328 microprocessor \\
\hline \multirow[t]{2}{*}{ Camera } & Pacific Co. VPC-895A \\
\hline & CCD1/3 PAL-25-fps \\
\hline Depth sensor & Pressure Sensor MS5803-14BA \\
\hline Sampling period & $40 \mathrm{~ms}$ \\
\hline \multirow[t]{3}{*}{ Surface computer } & Dell Latitude E6230- Intel Core i7 -2.9 GHz \\
\hline & Windows 7 Professional 64 bits \\
\hline & Microsoft Visual C ++2010 \\
\hline Tether length & $150 \mathrm{~m}$ \\
\hline
\end{tabular}

\subsection{Proposed Experimental Scenarios}

To test the robustness of the proposed control scheme, four different scenarios have been performed, namely:

(i) Scenario 1: Nominal case.

In this scenario the underwater vehicle follows a predefined desired trajectory in depth and yaw in the absence of external disturbances. During this test, the controller's feedback gains are adjusted to obtain the best tracking. These gains remain unchanged during the rest of the experiments. 
Table 2: Control gains used in real-time experiments

\begin{tabular}{rrrr} 
Depth & $b_{p 3}=20$ & $d_{p 3}=0.05$ & $u_{p 3}=0.1$ \\
& $b_{i 3}=15$ & $d_{i 3}=0.25$ & $u_{i 3}=0.2$ \\
$b_{d 3}=13$ & $d_{d 3}=0.25$ & $u_{d 3}=0.2$ \\
Yaw & $b_{p 6}=4.5$ & $d_{p 6}=0.015$ & $u_{p 6}=0.2$ \\
& $b_{i 6}=1$ & $d_{i 6}=0.015$ & $u_{i 6}=0.2$ \\
$b_{d 6}=0.2$ & $d_{d 6}=0.15$ & $u_{d 6}=0.2$ \\
\hline
\end{tabular}

(ii) Scenario 2: Robustness towards parametric uncertainties

In this test the buoyancy and damping of the vehicle are modified to test the effectiveness of the controller and its robustness towards parametric uncertainties.

(iii) Scenario 3: External disturbances rejection.

This test is inspired by a more realistic scenario, where the vehicle has the task of loading an object and when it reaches a certain depth, it drops that object. In this test we can observe a sudden change in the vehicle's weight and we can see how it affects the controller's performance.

(iv) Scenario 4: Comparison with other controllers. In this test, the performance of the NLPID controller is compared with the classical PD controller and the NLPD control in parametric robustness tests.

\subsection{Tuning the nonlinear PID controller}

The gains of the proposed controller have been tuned heuristically and following sequentially the steps below:

- $d_{p j}$ is chosen, taking into account that the interval $\left[-d_{p j}, d_{p j}\right]$ is the linear region of the proposed controller.

- Considering $b_{d j}=b_{i j}=0$ and $\mu_{p j}=0 ; b_{p j}$ is increased until the closedloop system oscillates. 
- $d_{i j}$ is chosen equal or bigger than $d_{p j}$, and $\mu_{i j}=0$.

- Then $b_{i j}$ is increased until the system oscillations decrease.

- $d_{d j}$ is chosen bigger than $d_{p j}$, and $\mu_{d j}=0$.

- $b_{d j}$ is increased until the system oscillations decrease.

- $\mu_{p j}, \mu_{d j}$ and $\mu_{i j}$ are adjusted to improve the system behavior, considering $\mu_{p j} \leq \mu_{d j}$ and $\mu_{p j} \leq \mu_{i j}$.

Finally, the control gains used through out the whole set of real-time experiments are summarized in Table 2 ,

\subsection{Scenario 1: Control in nominal conditions}

The upper plot of Figure 5 shows the depth and yaw controller performance of the robot during the first scenario. In this experiment, the vehicle follows a predefined trajectory in depth going from the surface to a maximal depth of $30 \mathrm{~cm}$, where the vehicle remains stable in that position for 20 seconds and finally emerges to $20 \mathrm{~cm}$ and hovers until the trial ends. For the yaw motion, the vehicle turns from its initial position of 60 degrees in 6 seconds. Then, it remains stable in that position for 20 seconds. Finally, the robot goes to -60 degrees and stays there until the test ends. It can be observed that the controller has a short lapse of time to converge to the reference trajectory with a slight tracking error, as seen in the error plot in the middle of Figure 5. This can be confirmed through numerical data from the Root Mean Square Error (RMSE), which is shown in Table 3 It is worth noting that oscillations (of an amplitude smaller than $1 \mathrm{~cm}$ ) that are perceived in the depth trajectory tracking, could be a consequence of the depth sensor's accuracy. Finally, the evolution of the control inputs is displayed at the bottom of Figure 5

\subsection{Scenario 2: Robustness towards parameter's uncertainties}

In order to evaluate the robustness of the proposed controller against parametric uncertainties, we changed the buoyancy of the vehicle by attaching buoys 
on both sides of the vehicle, thus increasing the floatability by $200 \%$. To modify the damping of the submarine, we attached a large rigid plastic sheet $(45 \times 10$ $\mathrm{cm}$ ) on one side of the submarine, thus increasing the rotational damping along z-axis by approximately $90 \%$ (see illustration in Figure 3 ).

The tracking trajectory for depth and yaw motion is shown on the top of Figure 6. The NLPID takes a short lapse of time to converge to the reference depth trajectory, it is due to the fact that the vehicle needs more energy to overcome the added buoyant force. Despite the big persistent disturbance, the controller is able to maintain a performance as good as in the nominal case. However, the yaw tracking trajectory is reduced when the vehicle turns, but maintains a good steady-state performance. The RMSE for both scenarios is given in Table 3, it can be noted that the values are very close to the ones observed in the nominal case. The tracking errors are shown in the middle of 285 Figure 6. In this figure, we can see the impact of the disturbances, the error increases when the vehicle submerges, emerges or turns. Finally, in the bottom of Figure 6, we can examine the evolution of the control inputs versus time. For example, for depth following, we can observe that the force increases almost twice as much when compared to the nominal case. This suggests that there is a strong compromise between the controller's ability to reject disturbances and the increase in energy that is demanded from the actuators.

\subsection{Scenario 3: Robustness towards external disturbances}

In some applications, underwater vehicles are equipped with robotic manipulators which allow them to carry or manipulate objects and take them to a specific location or pick them up from the ocean floor to transport them to the surface. This scenario is inspired by this practical case. To simulate this the robot carries a load, a metallic block of $1 \mathrm{~kg}$ has been attached to the submarine with a $20 \mathrm{~cm}$-long length of rope. In this scenario, the maximal depth of the reference trajectory has been set to $40 \mathrm{~cm}$. As the maximum depth of the pool is $50 \mathrm{~cm}$, the robot will be suddenly disturbed when it will reach 30 centimeters, because the metallic block will touch the floor, thus suddenly canceling its 


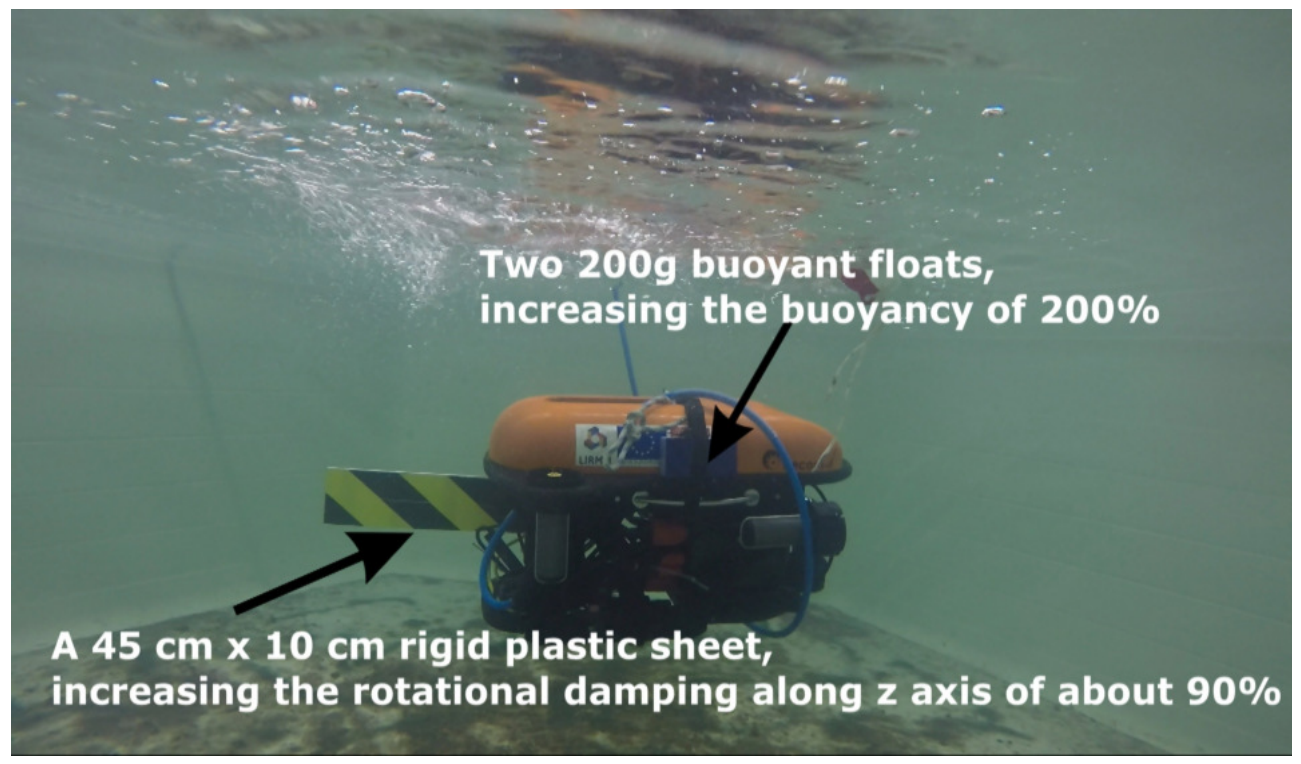

Figure 3: Leonard underwater vehicle with the two additional buoyant floats and a rigid plastic sheet, which increase the buoyancy and the damping along the $\mathrm{z}$ axis

weight. The disturbance will remain until the robot will move up and reach 30 $\mathrm{cm}$, where the extra weight will act again (Figure 4). This simulates both the sudden release and the recovery of a load by the robot.

The obtained results for this scenario are depicted in Figure 7. At the top of the graph, we can see the influence of the extra weight because it changes the initial position of the submarine to $30 \mathrm{~cm}$ deep. When the test begins, the robot converges to the desired trajectory in about 5 seconds. Two seconds later, the weight of the vehicle suddenly changes and the NLPID control compensates the effects of the disturbance some seconds later thanks to the integral term of the controller. When the vehicle moves up, the extra weight acts again on the submarine and reduces the trajectory tracking. Again, the NLPID controller compensates this disturbance as we can observe at the end of the graph. The error plots are displayed in the middle of Figure 7 , while the numerical value of the RMSE is given in Table 3 . The evolution of the control input is displayed at the bottom of Figure 7 . 


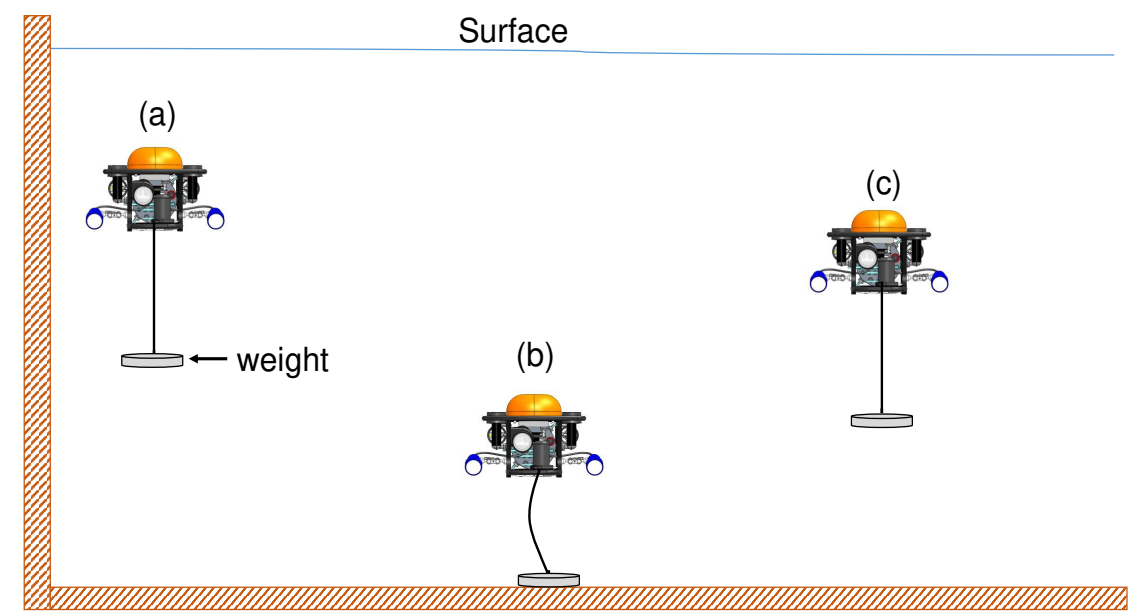

Figure 4: Description of controller robustness towards external disturbances test. A plate weighing $1 \mathrm{~kg}$ is attached to the robot as shown in (a). When the robot reaches $30 \mathrm{~cm}$, the influence of the weight disappears (b). Finally, when the robot moves towards the surface, the influence of the weight acts on the robot again (c). 
Table 3: Root Mean Square Error for NLPID controller for trajectory tracking .

\begin{tabular}{ccc} 
Case & $R M S E_{z}(m)$ & $R M S E_{\psi}(\mathrm{deg})$ \\
\hline Nominal & 0.0009 & 0.0265 \\
Parametric uncertainties & 0.0017 & 0.4269 \\
External disturbances & 0.0030 & 0.0605 \\
\hline
\end{tabular}

\subsection{Scenario 4: Comparison to other controllers}

To highlight the improvements of our proposed NLPID controller over standard methods, the NLPID is compared to the well-known PD with buoyancy disturbance.

In the middle of Figure 8 , the plot of errors are depicted and the improvement of each controller is visually obvious and can be confirmed numerically through the RMSE in Table 4. Finally, the control inputs are displayed at the bottom 340 of Figure 8. It is worth noting that at the beginning of the test, the NLPID 
demands more energy than the other controllers, but this energy reduces quickly as the NLPID reaches the reference trajectory.

Table 4: Root Mean Square Error comparison for PD, NLPD and NLPID controllers.

\begin{tabular}{ccc}
\hline Controller & $R M S E_{z}(m)$ & $R M S E_{\psi}(\mathrm{deg})$ \\
\hline PD & 0.0789 & 0.5500 \\
NLPD & 0.0374 & 0.3371 \\
NLPID & 0.0017 & 0.4269 \\
\hline
\end{tabular}

\section{Conclusion}

In this paper, a decoupled nonlinear PID (NLPID) control has been develfor example. 

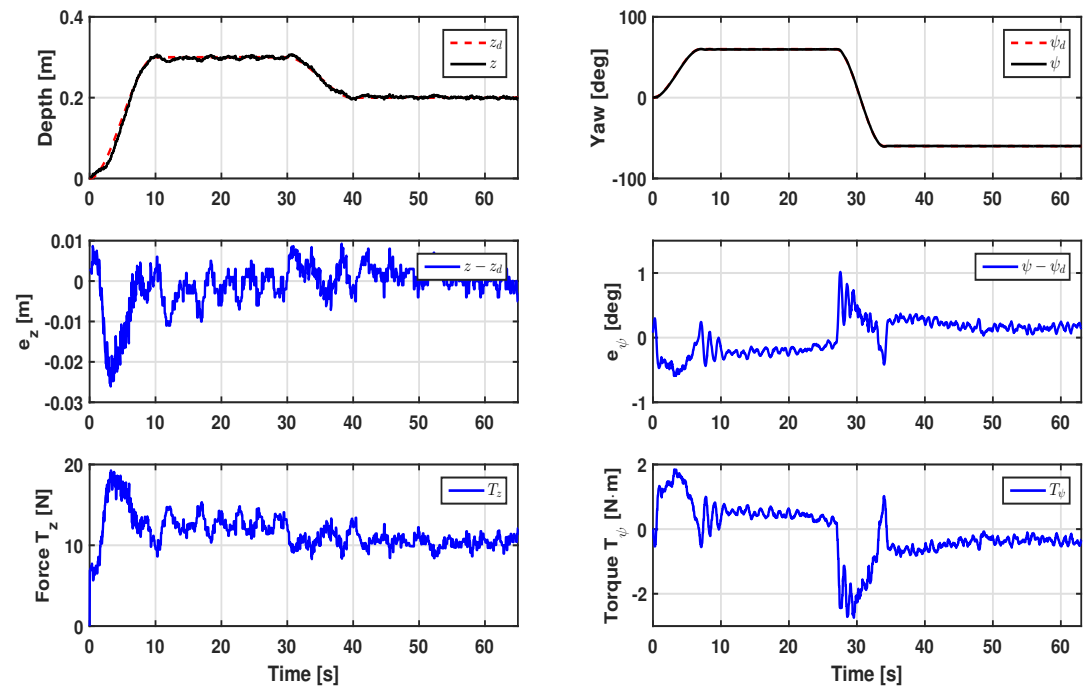

Figure 5: Performance of the NLPID controller nominal design. (Upper) Trajectory tracking in depth and yaw in absence of disturbances. (Middle) Plots of the error signal. (Lower) Evolution of the control inputs.

\section{Acknowledgment}

This work was supported by Conacyt, grant 490978. The Leonard underwater vehicle has been financed by the European Union (FEDER grant $\mathrm{n}^{\circ} 49793$ ) and the Region Occitanie (ARPE Pilot Plus project). The authors would like to express their gratitude to the anonymous reviewers for their comments for the improvement of the manuscript. 

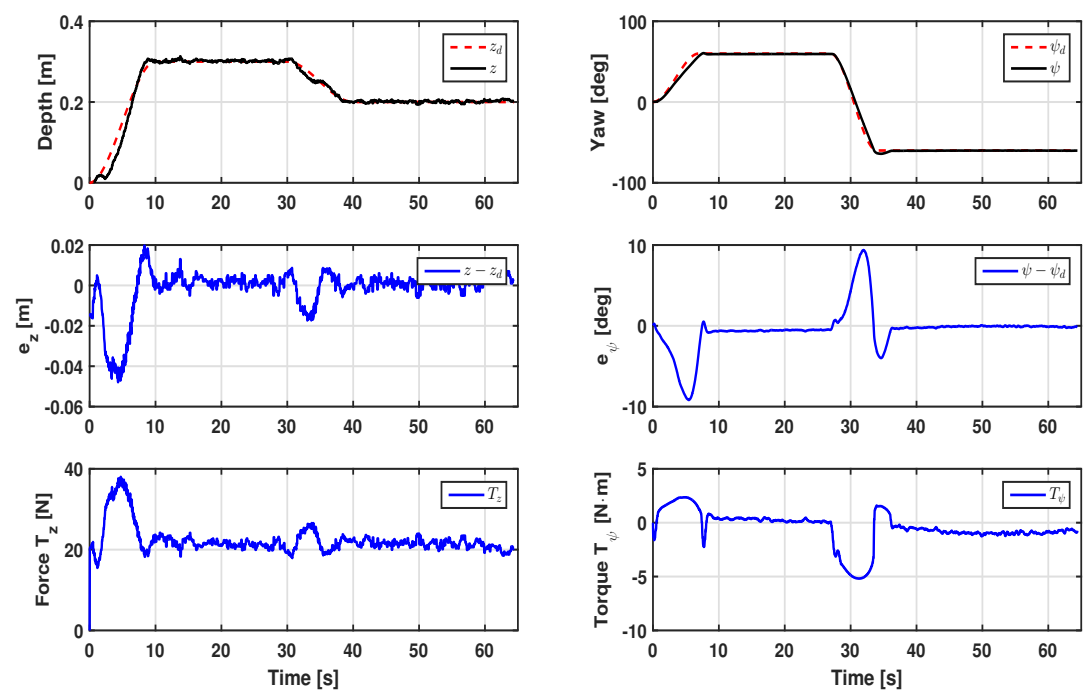

Figure 6: Robustness NLPID controller performance towards parametric uncertainties. The floatability of the submarine was increased by $200 \%$ while the damping along the z-axis was modified by up to $90 \%$ when compared to the nominal case. 

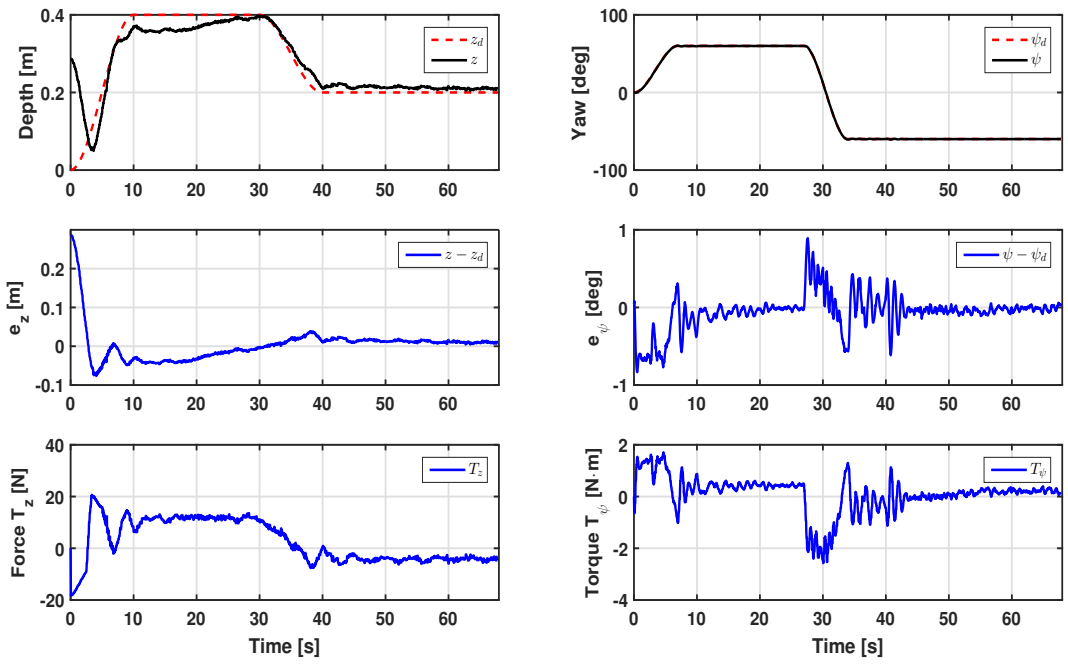

Figure 7: NLPID controller performance under external disturbance test. A $1 \mathrm{~kg}$ weight is attached to the robot and when the vehicle reaches a depth of $30 \mathrm{~cm}$ the influence of the weight disappears. Finally, when the robot moves towards the surface, the action of the weight disturbs the robots dynamics again.
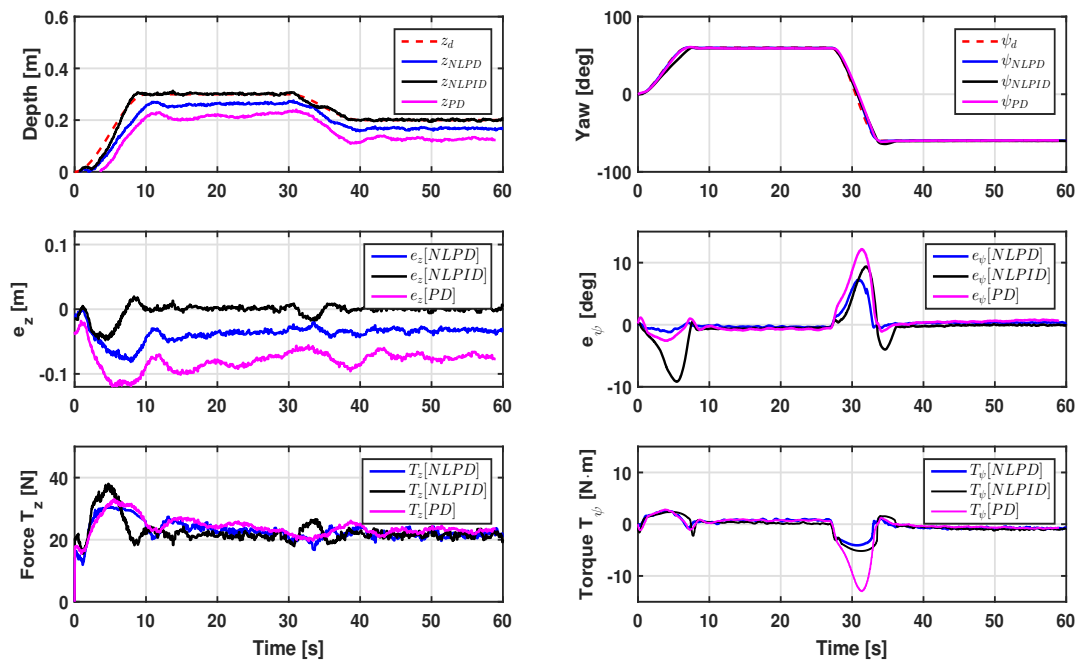

Figure 8: NLPID, NLPD and PD controller performance comparison towards parametric uncertainties. The floatability of the submarine was increased by $200 \%$ while the damping along the z-axis was modified by up to $90 \%$ when compared to the nominal case. 


\section{References}

370

[1] S. Zhao, J. Yuh, Experimental study on advanced underwater robot control, IEEE transactions on robotics 21 (4) (2005) 695-703.

[2] B. Jalving, The ndre-auv flight control system, IEEE Journal of Oceanic Engineering 19 (4) (1994) 497-501.

[3] T. I. Fossen, Guidance and control of ocean vehicles, John Wiley \& Sons

[4] P. Herman, Decoupled pd set-point controller for underwater vehicles, Ocean Engineering 36 (6-7) (2009) 529-534.

[5] N. Radmehr, H. Kharrati, N. Bayati, Optimized design of fractional-order pid controllers for autonomous underwater vehicle using genetic algorithm, in: Electrical and Electronics Engineering (ELECO), 2015 9th International Conference on, IEEE, 2015, pp. 729-733.

[6] L. Zhang, L. Zhang, S. Liu, J. Zhou, C. Papavassiliou, Low-level control technology of micro autonomous underwater vehicle based on intelligent computing, Cluster Computing (2018) 1-12.

385

[7] M. H. Khodayari, S. Balochian, Modeling and control of autonomous underwater vehicle (auv) in heading and depth attitude via self-adaptive fuzzy pid controller, Journal of Marine Science and Technology 20 (3) (2015) $559-578$.

[8] M. M. Hammad, A. K. Elshenawy, M. El Singaby, Trajectory following 390 and stabilization control of fully actuated auv using inverse kinematics and self-tuning fuzzy pid, PloS one 12 (7) (2017) e0179611.

[9] X. Xiang, C. Yu, Q. Zhang, Robust fuzzy 3d path following for autonomous underwater vehicle subject to uncertainties, Computers \& Operations Research 84 (2017) 165-177. 
[10] R. Rout, B. Subudhi, Inverse optimal self-tuning pid control design for an autonomous underwater vehicle, International Journal of Systems Science 48 (2) (2017) 367-375.

[11] Y. Zhai, L. Liu, W. Lu, Y. Li, S. Yang, F. Villecco, The application of disturbance observer to propulsion control of sub-mini underwater robot, in: International Conference on Computational Science and Its Applications, Springer, 2010, pp. 590-598.

[12] Y. Shen, K. Shao, W. Ren, Y. Liu, Diving control of autonomous underwater vehicle based on improved active disturbance rejection control approach, Neurocomputing 173 (2016) 1377-1385.

[17] P. Sarhadi, A. R. Noei, A. Khosravi, Adaptive \mu $\mu$-modification control for a nonlinear autonomous underwater vehicle in the presence of actuator saturation, International Journal of Dynamics and Control 5 (3) (2017) 596-603.

420

[13] N. Q. Hoang, E. Kreuzer, Adaptive pd-controller for positioning of a remotely operated vehicle close to an underwater structure: Theory and experiments, Control Engineering Practice 15 (4) (2007) 411-419.

[14] Y. C. Sun, C. C. Cheah, Adaptive control schemes for autonomous underwater vehicle, Robotica 27 (1) (2009) 119-129.

[15] E. Campos, A. Chemori, V. Creuze, J. Torres, R. Lozano, Saturation based nonlinear depth and yaw control of underwater vehicles with stability analysis and real-time experiments, Mechatronics 45 (2017) 49-59.

[16] P. Sarhadi, A. R. Noei, A. Khosravi, Model reference adaptive pid control with anti-windup compensator for an autonomous underwater vehicle, Robotics and Autonomous Systems 83 (2016) 87-93.

M. Kim, H. Joe, J. Pyo, J. Kim, H. Kim, S.-c. Yu, Variable-structure pid controller with anti-windup for autonomous underwater vehicle, in: Oceans-San Diego, 2013, IEEE, 2013, pp. 1-5. 
[19] R. Kelly, R. Carelli, A class of nonlinear pd-type controllers for robot manipulators, Journal of Field Robotics 13 (12) (1996) 793-802.

[20] J. L. Meza, V. Santibáñez, R. Soto, M. A. Llama, Fuzzy self-tuning pid semiglobal regulator for robot manipulators, IEEE Transactions on industrial electronics 59 (6) (2012) 2709-2717.

[21] L. G. García-Valdovinos, T. Salgado-Jiménez, M. Bandala-Sánchez, L. Nava-Balanzar, R. Hernández-Alvarado, J. A. Cruz-Ledesma, Modelling, design and robust control of a remotely operated underwater vehicle, International Journal of Advanced Robotic Systems 11 (1) (2014) 1.

[22] M. Karkoub, H.-M. Wu, C.-L. Hwang, Nonlinear trajectory-tracking control of an autonomous underwater vehicle, Ocean Engineering 145 (2017) 188 - 198. doi:https://doi.org/10.1016/j.oceaneng.2017.08.025. URL http://www.sciencedirect.com/science/article/pii/ S0029801817304754

[23] S. of Naval Architects, M. E. U. Technical, R. C. H. Subcommittee, Nomena clature for Treating the Motion of a Submerged Body Through a Fluid: Report of the American Towing Tank Conference, Technical and research bulletin, Society of Naval Architects and Marine Engineers, 1950. URL https://books .google.com.mx/books?id=sZ_bOwAACAAJ

[24] K. D. Do, J. Pan, Control of ships and underwater vehicles: design for underactuated and nonlinear marine systems, Springer Science \& Business Media, 2009. 


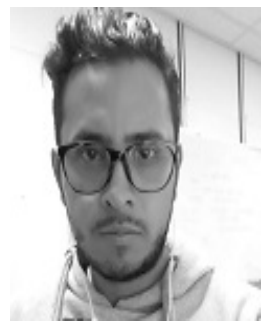

455

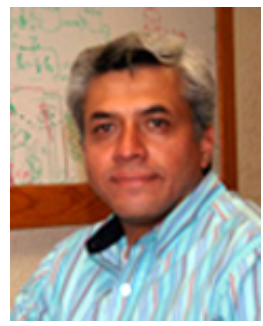

control of mini-submarines.

460

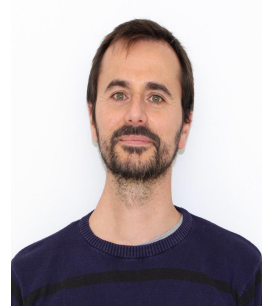

ter computer vision

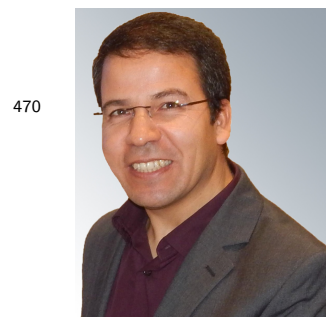

Jesus GUERRERO received his Ph.D. degree in 2019 in automatic control from the Center for Research and Advanced Studies of the National Polytechnic Institute (CINVESTAV), Mexico. His research interests include nonlinear, adaptive and time-delay control and their applications in underactuated systems, ground, aerial, and underwater vehicles.

Jorge TORRES was born in Mexico City, on May 13, 1960. He received his Ph.D. degree in Automatic Control from LAG, INPG, France, in 1990. He joined the Department of Electrical Engineering at the CINVESTAV, Mexico, in 1990. His research interest lies in the structural approach of linear systems, stability of multivariate polynomials, and the control of bioprocess for waste water treatment and the Vincent CREUZE received his Ph.D. degree in 2002 in robotics from the University Montpellier 2, France. He is currently an associate professor at the University Montpellier 2, attached to the Robotics Department of the LIRMM (Montpellier Laboratory of Computer Science, Robotics, and Microelectronics). His research interests include design, modelling, and control of underwater robots, as well as underwa-

Ahmed CHEMORI received his M.Sc. and Ph.D. degrees respectively in 2001 and 2005, both in automatic control from the Grenoble Institute of Technology. Then, he has been a Post-doctoral fellow for one year with the Automatic control laboratory of Grenoble. He is currently a tenured research scientist in Automatic control and Robotics at the 
Montpellier Laboratory of Informatics, Robotics, and Microelectronics. His research interests include nonlinear, adaptive and predictive control and their applications in humanoid robotics, underactuated systems, parallel robots, and underwater vehicles.

Eduardo CAMPOS received his B.S. degree in elec-

480

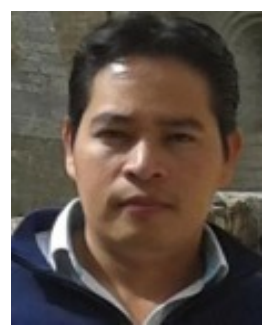

tromechanical engineering from the ITZ (Instituto Tecnolgico de Zacatepec) in 2008, and the M.S. degree in automatic control from the CINVESTAV (Centro de Investigación y de Estudios Avanzados del IPN), México, in 2010. He received his Ph.D. degree in 2014 from CINVESTAV and LIRMM (Laboratoire dInformatique, de Robotique et de Microlectronique de Montpellier). Currently he is working on the development of the AUV (Autonomous Underwater Vehicle) and artificial vision applications in underwater robots. 